\title{
II. On the Reduction of a continued fraction to a series
}

\section{A. De Morgan}

To cite this article: A. De Morgan (1844) II. On the Reduction of a continued fraction to a series, Philosophical Magazine Series 3, 24:156, 15-17, DOI: 10.1080/14786444408644791

To link to this article: http://dx.doi.org/10.1080/14786444408644791

曲 Published online: 30 Apr 2009.

Submit your article to this journal $\pi$

Џ Article views: 4

Q View related articles $\sqsubset$ 
librio? These questions might be investigated with profit were not this communication already too extended.

44. It is proper to state, however, that DeCandolle's theory of the bending of plants towards light has been fully disproved, inasmuch as it is an effect due to the indigo rays, which have not power to decompose carbonic acid and produce lignin, \&c. (Mém. Soc. d'Arcueil, 1809, p. 104).

In conclusion, it appears that the following facts have been established :-

1st. That chlorophyl is produced by the more luminous rays, the maximum being in the yellow.

2nd. This formation is due to pure LIGHT, an imponderable distinct from all others.

3rd. That the ray towards which plants bend occupies the indigo space of Fraunhofer.

4.th. 'This movement is due to pure LIGHT, as distinguished from heat and tithonicity.

5th. 'That pure LIG H'T is capable of producing changes rehich result in the development of palpable motion.

6th. The bleaching of chlorophyl is most active in those parts of the spectrum which possess little influence in its production, and are complementary to the yellow rays.

7 th. This action is also due to pure LIGHT.

We have, therefore, an analysis of the action of every ray in the luminous spectrum upon vegetation. The several effects produced are not abruptly terminated within the limits of any of the spaces, but overlap to a certain extent, a fact which coincides with our experience of the properties of the rays. Whilst heat and tithonicity are capable of causing the union of mineral particles, light appears to be the only radiant body which rules pre-eninent in the organic world. To the animating beams of the sun we owe whatever products are necessary to our very existence.

New York, October 14, 1843.

II. On the Reduction of a Continued Fraction to a Series. By A. De Morgan, Professor of Mathematics in Univer. sity College, London*.

THE mode of reducing a continued fraction to a series has not received much attention, but as every specimen of law of development may contain useful hints, the following investigation will perhaps interest the mathematical reader.

It is required to develope into a series of powers of $x$ the continued fraction

* Communicated by the Author. 


$$
\frac{a_{1}}{b_{1}+} \frac{a_{2} x}{b_{2}+} \frac{a_{3} x}{b_{3}+}, \& c ., \quad \text { or } \frac{a}{1+} \frac{b x}{1+} \frac{c x}{1+}, \text { \&c., }
$$

to the second of which the first may be easily reduced. In reasoning it may be proper to use $a_{1}, a_{2}, a_{3}, \& c$. ; in working, $a, b, c, \& c$. will be found more convenient. If

$$
\mathrm{A}_{n}=\frac{a_{n}}{1+} \frac{a_{n+1} x}{1+}, \& \mathrm{c} ., \quad \mathrm{A}_{n+1}=\frac{a_{n+1}}{1+} \frac{a_{n+2} x}{1+}, 8 \mathrm{c} .
$$

we have $A_{n}\left(1+A_{n+1} x\right)=a_{n}$. If then $f\left(a_{m+1}, a_{n+1}, \& c.\right)$ be called the advanced form of $f\left(a_{m}, a_{n}, 8 \mathrm{c}\right.$. $)$, and if $\mathrm{A}$ be taken to be $P_{0}+P_{1} x+P_{2} x^{2}+, \& c$.; and if $Q_{0}, Q_{1}$, \&c. be the advanced forms of $\mathrm{P}_{0}, \mathrm{P}_{1}$, \&c., we have, from the equation between $A_{1}$ and $A_{2}$,

$$
\begin{aligned}
\mathbf{P}_{0} & =a_{1}, \mathbf{P}_{1}=\mathrm{P}_{0} \mathrm{Q}_{0}, \mathrm{P}_{2}=\mathrm{P}_{0} \mathrm{Q}_{1}+\mathrm{P}_{1} \mathrm{Q}_{0}, \& \mathrm{c} . \\
\mathbf{P}_{n+1} & =\mathrm{P}_{0} \mathrm{Q}_{n}+\mathrm{P}_{1} \mathbf{Q}_{n-1}+\ldots+\mathrm{P}_{n-1} \mathbf{Q}_{1}+\mathrm{P}_{n} \mathrm{Q}_{0}
\end{aligned}
$$

which gives an easy law of formation for a few terms. Thus we have, using $a, b, \&$ c. for $a_{1}, a_{2}, \& c$.,

$$
\begin{aligned}
\mathbf{P}_{0}=a, \mathrm{P}_{1}=a b, \mathrm{P}_{2}=a(b c)+a b(b)=a b c+a b^{2} \\
\mathbf{P}_{3}=a\left(b c d+b c^{2}\right)+a b(b c)+\left(a b c+a b^{2}\right) b, \\
=a b c d+a b c^{2}+2 a b^{2} c+a b^{3}, \\
\mathbf{P}_{4}=a b c d e+a b c d^{2}+2 a b c^{2} d+a b c^{3}+2 a b^{2} c d \\
\quad+3 a b^{2} c^{2}+3 a b^{3} c+a b^{4} .
\end{aligned}
$$

The results of this method would give little encouragement to attempt finding the law of these terms, which is, however, very simple, as follows: let such an expression as $a b^{\beta} c^{\gamma} d^{\gamma} \ldots$ in which the order $a, b, c, d, \& c$. is unbroken, be called consecutive; and let $m_{n}$ denote the coefficient of the $m$ th power of $x$ in the development of $(1+x)^{n}$; then will the coefficient $P_{i}$ be

$$
\Sigma \gamma_{\beta} \delta_{\gamma} \varepsilon_{\delta} \ldots \times a b^{\beta} c^{\gamma} d^{\delta} e^{\varepsilon} \ldots
$$

the sign $\Sigma$ extending to every way in which $\beta+\gamma+\delta+\ldots$ $=i$, on condition only that every term shall be consecutive, that is, that no one of the set $\beta, \gamma, \& c$. shall vanish, unless all the subsequent ones vanish also. "This law will be evident on a very slight consideration of another mode of development, namely, that of $a \div(1+b x)$, followed by the substitution of $b \div(1+c x)$ for $b$, followed by that of $c \div(1+d x)$ for $c$, and so on.

The number of terms in $P_{i}$ must be $2^{i-1}$, since they are formed by writing over $b, c, d$, \&c. exponents $\beta, \gamma, \& c$. in every possible way and order in which $i$ can be $\beta+\gamma+\ldots$ 
without any one of the set $\beta, \gamma$, \&c. being nothing. The sum of the coefficients is half the coefficient of $x^{i+1}$ in $\sqrt{ }(1+4 x)$, as is easily proved.

The readiest way of forming $P_{i+1}$ from $P_{i}$ is as follows:(1.) Put on the next letter to every term of $P_{i}$, and also repeat the last letter of each term once more than it occurs already; thus $a b^{2} c$ gives $a b^{2} c d+a b^{2} c^{2}$. (2.) Put all the results of (1.) together, and correct or introduce coefficients by the law above ascertained.

It is also worth notice that the portions of $P_{i}$ which do not contain any letters beyond a given one follow the law of a recurring series. 'Thus, if $\mathrm{P}_{n, k}$ signify all that portion of $\mathrm{P}_{n}$ in which nothing beyond $k$.occurs, we have

$$
\begin{aligned}
\mathrm{P}_{n, b}= & b \mathrm{P}_{n-1, b}, \mathrm{P}_{n, c}=(b+c) \mathrm{P}_{n-1, c} \\
& \mathrm{P}_{n, d}=(b+c+d) \mathrm{P}_{n-1, d}-b d \mathrm{P}_{n-2, d},
\end{aligned}
$$

and so on; the coefficients $(b+c+d)$, \&c. being derived from the denominators of the ordinary approximations.

The mode thus given of turning a continued fraction into a series is not so easy in practice as the one derived from inverting the process of turning the ratio of two infinite series into a continued fraction; but the law is worth consideration, the more especially as, from the presence of none but consecutive terms, it cannot be directly connected with Arbogast's methods. 'This is the reason why the reader looks in vain for anything about continued fractions in the Calcul des Derivations. The most complete account I know of continued fractions is in Eytelwein's Grundlehren, \&c., in which, however, the inverse method occupies only six pages, the direct one eighty-nine; and no general law is given for the series considered in this paper.

III. On the Action of Allalies on Wax. By RoBERT Warington and W. Francis, Esq.*

FEW subjects have of late engaged so much the attention of chemists as that relative to the formation of fat in the animal organization, a subject fraught with results of the highest importance both to science and its applications to rural œconomy.

Twotheories have been proposed to account for its origin :1843.

* Communicated by the Chemical Society; having been read May 16 Phil. Mag. S. 3. Vol. 24. No. 156. Jan. 1844. 